\title{
Quantitative microstructure and defect density analysis of polycrystalline tungsten reference samples after different heat treatments
}

\author{
A. Manhard*, M. Balden, S. Elgeti \\ Max-Planck-Institut für Plasmaphysik, Boltzmannstr. 2, D-85748 Garching, Germany \\ * corresponding author: armin.manhard@,ipp.mpg.de; \\ phone: +49 (0) 8932991025 fax: +49 (0) 8932991212
}

\begin{abstract}
$\underline{\text { Abstract }}$
In order to provide a solid basis for the correlation of microstructure and hydrogen isotope retention in tungsten, reference samples with different microstructures were prepared from a single batch of polycrystalline tungsten by standardised polishing and heat treatment procedures. Representative samples were analysed by scanning electron microscopy and scanning transmission electron microscopy as well as by electron backscatter diffraction. We show that if the annealing temperature is increased from 1200 to $1500 \mathrm{~K}$, practically only the density of dislocations and grain boundaries with very small misorientations of less than $2^{\circ}$ is reduced, while for annealing at 1700 and $2000 \mathrm{~K}$, also the density of high-angle grain boundaries is reduced due to grain growth. Furthermore, the dislocation density is reduced by nearly two orders of magnitude compared to tungsten annealed at $1200 \mathrm{~K}$. We also comment on two different textures on the front and rear side of the samples that were observed both by $\mathrm{X}$-ray diffraction and EBSD.
\end{abstract}

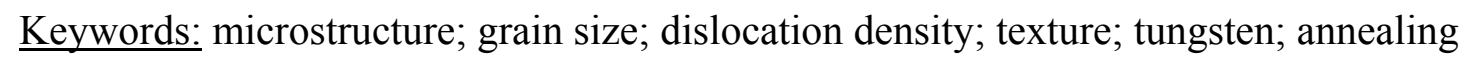

PACS: 61.05.cp (XRD); 61.05.J-(Electron diffraction and scattering); 61.72.Ff (defect imaging by electron microscopy); 61.72.Lk (dislocations); 61.72.Mm (grain boundaries); 81.05.Bx (Metals); 81.10.Jt (recrystallization); 81.40.Ef (annealing) 


\section{Introduction}

Tungsten is a promising candidate material for the plasma-facing surfaces in a future fusion reactor. One of the favourable properties of tungsten for this application is its low interstitial solubility and retention for hydrogen isotopes $[1,2]$. Particularly for the radioactive fusion fuel tritium, a low inventory in the plasma-facing walls is important because of nuclear safety as well as from economic considerations, namely the goal of tritium self-sufficiency of such a reactor.

The low interstitial solubility of hydrogen isotopes in tungsten also means that the main contribution to the total retained inventory of, e.g., deuterium after exposure to a plasma is typically due to deuterium trapped at crystal defects such as vacancies, impurity atoms, dislocations or grain boundaries (see, e.g., [3]). For studying such trapping effects in detail, it is necessary to quantify the defect densities in tungsten samples before exposing them to a deuterium plasma.

The density of grain boundaries can be derived from scanning electron micrographs, where (for a pure material such as the tungsten investigated here) regions with different backscatter electron (BSE) contrast can be assigned to (sub)grains. Orientation maps by electron backscatter diffraction yield complementary information. Both methods have their individual strengths and weaknesses, which will be discussed in this article. Dislocations are easily visible by (scanning) transmission electron microscopy, so their density can be estimated from image analysis as well. Unfortunately, point defects such as vacancies can usually not be detected by electron microscopy.

In this article, a systematic study of the densities of dislocations and grain boundaries in tungsten reference samples after different heat treatments is presented. Hot-rolled, polycrystalline tungsten samples with dimensions $15 \times 12 \times 0.8 \mathrm{~mm}^{3}$ and with a specified purity 
of 99.97 wt.\% were used as a base material. The samples were produced by Plansee SE, Reutte, Austria. According to the manufacturer, the deformation degree exceeded $60 \%$. The rolling direction was parallel to the short edges of the investigated samples. All samples were from one single manufacturing batch and had received a coarse surface finish by the manufacturer to ensure plane-parallel surfaces and a constant thickness. The as-received samples were polished to a metallographic finish with a standardised procedure [4], and subsequently annealed at different temperatures in order to modify the microstructure. Thereafter, they were analysed by scanning electron microscopy (SEM) and scanning transmission electron microscopy (STEM) in order to determine the densities of grain boundaries and dislocations. Electron backscatter diffraction (EBSD) was used to statistically classify the types of grain boundaries in the samples by their misorientation angle. In addition to texture analysis by EBSD, X-ray diffraction (XRD) was employed to investigate the textures on the surfaces of a larger number of samples.

The results presented here serve as the basis for systematically investigating the dependence of hydrogen isotope retention on the densities of different defects in tungsten. First results using exactly this material with the same surface finish and heat treatments, but with a less detailed characterisation, were already published $[5,6,7,8]$.

\section{Heat treatment}

Heat treatment of the samples was performed either at $1200 \mathrm{~K}$ for 60 minutes, or at 1500 , 1700 or $2000 \mathrm{~K}$ for 30 minutes each. The $1200 \mathrm{~K}$ treatment was performed in a high-vacuum $\left(\sim 10^{-4} \mathrm{~Pa}\right)$ oven whose hot zone is composed entirely of molybdenum. This treatment is supposed to result in stress relief of the sample, and also to reduce the initial protium $\left({ }^{1} \mathrm{H}\right)$ content, which is introduced during the manufacturing process. In addition, this heat treatment 
is also sufficient to remove thick oxide layers at the sample surface. In a previous, less detailed study it was already found that annealing at $1200 \mathrm{~K}$ does not visibly change the grain structure of the sample [5]. Finally, $1200 \mathrm{~K}$ is also the typical maximum temperature that occurs during analysis of the deuterium inventory after plasma exposure by thermal desorption spectroscopy (see, e.g., [5]). We therefore consider samples annealed under these conditions as a suitable reference both for this study and for deuterium loading experiments.

For the higher temperatures, a graphite oven with a protective gas atmosphere at an overpressure of $150 \mathrm{kPa}$ (Ar for 1500 and $1700 \mathrm{~K}$, He for $2000 \mathrm{~K}$ ) was used. In order to prevent the formation of carbides at the sample surface, the samples were enclosed either in a tantalum container blocking all direct lines of sight to the surrounding graphite components, or wrapped in tungsten foils. According to literature [9], the recrystallization threshold of tungsten (i.e., the temperature at which full recrystallization takes place within one hour) is of the order of $1500 \mathrm{~K}$. Correspondingly, annealing at this temperature for only 30 minutes can be expected to produce a tungsten sample that is only partially recrystallized. For temperatures of $1700 \mathrm{~K}$ and above, the annealing time of $30 \mathrm{~min}$ is more than sufficient for full recrystallization [9], and the heat treatment also leads to substantial grain growth.

\section{Analysis techniques}

\subsection{BSE image analysis}

For the analysis of grain boundary densities, scanning electron micrographs with backscattered electron (BSE) contrast were used. The images were acquired with an annular solid-state backscatter electron detector in a Philips XL30 ESEM microscope. Typical images for all four annealing temperatures investigated here can be found in Figure 1, images a, c, e and $g$. For the analysis, the images were first segmented along the grain boundaries by hand. 
The resulting cells in the segmented image (i.e., the grains) were then analysed statistically with respect to their area $A_{\text {grain. }}$. From this, the corresponding equivalent square side length $a_{B S E}=A_{\text {grain }}{ }^{1 / 2}$ ("grain size") was derived as a linear measure of the grain size. The detailed grain size histograms are given in Figure 2. Accordingly, we calculate the average equivalent square side length as $\left\langle a_{B S E}\right\rangle=\left\langle A_{\text {grain }}{ }^{1 / 2}>\right.$. The average grain boundary area per unit volume $<S_{V}>$ ("grain boundary density") was estimated using a well-proven metallographic method [10]: On a metallographic section, it is related to the average number of intercepts per length $<N_{l}>$ of grain boundaries with an arbitrary straight line:

$$
<S_{V}>=2<N_{l}>
$$

Since $<N_{l}>$ is in turn given by $<N_{l}>=<A_{\text {grain }}>^{-1 / 2},<S_{V}>$ can be written also as

$$
<S_{V}>=2<A_{\text {grain }}>^{-1 / 2} \text {. }
$$

Note that here, in contrast to $\left\langle a_{B S E}\right\rangle$, the square root of the average is necessary in order to maintain area truth, and not the average of the square roots. In order to distinguish $\left\langle S_{V}\right\rangle$ derived from BSE images from $\left\langle S_{V}>\right.$ values derived from EBSD measurements (see section 3.3), we write $<S_{V, B S E}>$ from here on.

All error margins for grain sizes and grain boundary densities stated in this paper are derived directly from the statistical evaluation, i.e., from the standard deviations of the average values.

The BSE contrast mode does not allow distinguishing between small and high-angle grain boundaries. In the analysis presented here, the grain size statistics therefore also include both types of grain boundaries. A more detailed analysis of the types of grain boundaries was performed by electron backscatter diffraction and is described in section 3.3. 


\subsection{STEM image analysis}

Scanning transmission electron micrographs were acquired with a FEI Helios NanoLab 600 microscope with an acceleration voltage of up to $30 \mathrm{kV}$. The microscope is equipped with a retractable solid state STEM detector. It consists of a bright field (BF), an annular dark field (DF) and a 12-segment high-angle dark field (HADF) detector. The HADF detector can be used as a high-angle annular dark field (HAADF) detector. In many cases, however, it turned out that the contrast of dislocations could be improved by using a suitable selection of typically 3 adjacent segments (HADF-P mode).

The tungsten TEM samples were prepared by cutting disks with a diameter of $3 \mathrm{~mm}$ from heat-treated bulk samples by spark erosion. After pre-thinning by mechanical grinding, they were electropolished using a $15 \mathrm{~g} / \mathrm{l}$ aqueous $\mathrm{NaOH}$ solution. The samples were first electropolished from one side for approximately half of the time necessary for a perforation to occur. Then they were flipped around and electropolished from the other side until a small perforation (typical diameter $30-50 \mu \mathrm{m}$ ) was detected by an electro-optical circuit. Due to this preparation process, we estimate that the transparent area is approximately $0.2-0.4 \mathrm{~mm}$ away from either sample surface. We therefore consider all our STEM samples as being characteristic for the bulk of the material, rather than for the immediate near-surface regions.

Dislocation densities were derived from BF-STEM images because these contained the least amount of noise. Examples for all four annealing treatments can be found in Figure 1, images $\mathrm{b}, \mathrm{d}, \mathrm{f}$ and $\mathrm{h}$. Since the detector allowed simultaneous acquisition on multiple channels, DF and/or HADF-P images were also acquired and used for consistency checks. Dislocation densities were measured by tracing all dislocations in an image by hand. It turned out that semi-automatic image filtering (e.g., by edge enhancement) was just as time consuming and often less accurate due to noise in the image. In the resulting binary image, first the total projected length of all dislocations in the image was calculated. Due to limitations of our 
microscope system, we were not able to perform systematic tilting and orienting experiments. But due to the high contrast of the solid-state detector at $30 \mathrm{keV}$ primary electron energy, we assume that most dislocations were visible at least with a residual contrast, even if they were close to their respective extinction condition. Still, we cannot exclude that some dislocations in the observed sample regions were not visible, so the dislocation densities derived here should accordingly be considered as a lower limit for the true dislocation density.

Dislocation tangles, respectively the constituent dislocations of low-angle grain boundaries (if they could be resolved) were intentionally also counted. The dislocation density (in $\mathrm{m} / \mathrm{m}^{3}$ ) was then derived by dividing the average projected dislocation length per image area (in $\mathrm{m} / \mathrm{m}^{2}$ ) through the average sample thickness. Unfortunately, in the microscope used here, conventional methods for estimating the sample thickness, such as the comparison of elastic and inelastic contributions to an EELS spectrum or tilting experiments, could not be performed. Therefore, the sample thickness was estimated using the assumption that a clear diffraction contrast (S)TEM image is only obtained if the sample thickness is of the order of one or two elastic mean free paths of the probe electrons. Taking into account the electron energy of $30 \mathrm{keV}$, the acceptance angle of the bright field detector of $\sim 7.1^{\circ}$ and the NIST database on elastic electron scattering [11], the mean free path is about $22 \mathrm{~nm}$. Considering furthermore that the sample thickness varies somewhat across the image, and images were typically acquired some distance away from the thin edge of the sample, an average thickness of about $35 \mathrm{~nm} \pm 50 \%$ was estimated. Due to the small field of view at the magnifications necessary for observing dislocations, the statistical relevance of a dislocation density measurement in a single image is not very good. To improve this, several images from different locations were evaluated for each sample. The resulting values given in this paper are the averages of each set of measurements. The error margins given for dislocation 
densities are derived from the statistical variation of the dislocation density measurements and the uncertainty of the sample thickness.

\subsection{EBSD analysis}

Orientation mapping by electron backscatter diffraction was also performed with the FEI Helios NanoLab 600 system. Here, the sample surface normal was tilted by $57^{\circ}$ with respect to the electron beam. For acquisition of the diffraction patterns, a HKL Nordlys II detector together with the Channel 5 acquisition and analysis software was used. EBSD maps were acquired on polished bulk samples that had gone through the different heat treatments described above in section 2 (see Figure 3). While the BSE contrast is very sensitive even on small misorientations between neighbouring grains, it does not allow distinguishing the misorientation angles. This information was derived from EBSD maps, which contain the full grain orientation information. For the grain structure reconstruction, non-solved pixels in the ESBD map were post-processed using the Channel 5 software. First, the "wild spike" removal routine was executed, and then the unsolved pixels were extrapolated with the "standard" filter using 5 neighbours. The initial orientation maps had fractions of non-solved pixels of $9.8 \%, 18 \%, 7.7 \%$ and $11 \%$ for the samples annealed at $1200,1500,1700$ and $2000 \mathrm{~K}$. After the post-processing described above, the corresponding non-solved fractions were $0.5 \%$, $2.2 \%, 2.5 \%$ and $1.1 \%$. An example comparing original and post-processed data can be found in Fig. 3d.

In order to distinguish grain boundaries from misorientation gradients as well as from small random fluctuations of the solution of the electron backscatter patterns, grain boundaries were defined as the loci of misorientations $\theta \geq 2^{\circ}$ between neighbouring pixels. The different grain boundaries within the mapping areas were classified as either low-angle for misorientation 
angles of $2^{\circ} \leq \theta<10^{\circ}$, respectively as high-angle for $\theta \geq 10^{\circ}$ (Figure $3 \mathrm{a}$ and $\mathrm{d}$ ). This is motivated by experiments of Liu and Shen [12], who investigated the fracture behaviour of tungsten bi-crystals with a twist boundary. Their analysis showed that a crack initiated perpendicular to the grain boundary bridges the boundary for small misorientations $\theta$. For increasing $\theta$, starting at about $10^{\circ}$, intergranular fracture starts to occur, i.e., the crack is deflected and follows the grain boundary. From the histograms of misorientations between neighbouring pixels on the EBSD maps, the fractions of low-angle grain boundaries with $2^{\circ} \leq$ $\theta<10^{\circ}$ and of high-angle boundaries with $\theta \geq 10^{\circ}$ were derived.

We also calculated statistics for the grain areas in the same manner that was already described in section 3.1. for the BSE images. The statistical evaluation was performed both for zones enclosed by any grain boundary with a misorientation of at least $2^{\circ}$, and for zones delimited only by high-angle boundaries (i.e., with $\theta \geq 10^{\circ}$ ). Also in analogy to the BSE image analysis, we again derived grain boundary densities $\left\langle S_{V}>\right.$ as well as equivalent square size lengths (i.e., grain sizes). We label the average grain sizes considering only high-angle boundaries with $\theta \geq 10^{\circ}\left\langle a_{10^{\circ}}\right\rangle$, and the grain size including all grain boundaries with $\theta \geq 2^{\circ}$ $\left\langle a_{2^{\circ}}\right\rangle$. The corresponding grain boundary densities are $\left\langle S_{V, 10^{\circ}}\right\rangle$ and $\left\langle S_{V, 2^{\circ}}\right\rangle$, respectively. The underlying grain size histograms and the obtained average values can be seen in Figure 2 together with the ones derived from BSE image analysis. Figures 3 and 4 compile the grain boundary densities and classification into small and high-angle grain boundaries for all annealing temperatures.

The EBSD maps were also evaluated with respect to the texture after heat treatment at the four different temperatures. In addition, some EBSD analyses on a metallographic prepared and electro-polished cross-section of a stress-relieved sample were performed (Figure 6 a-c). 


\subsection{X-ray diffraction}

In order to obtain statistical information on the textures of tungsten samples, we also performed X-ray diffraction (XRD) analysis on many stress-relieved as well as recrystallized samples. The samples were investigated on the front side and in several cases additionally on the back side. For this analysis, we used a Seifert XRD 3003 PTS X-ray diffractometer with a $\mathrm{Cu} \mathrm{K}$ source $(0.154 \mathrm{~nm})$. On several tens of samples, we acquired pole figures of the $\{200\}$, $\{100\},\{222\}$ and $\{211\}$ diffraction peaks of $\mathrm{W}$ for a detailed look at the textures. For analysing quickly a large number of additional samples, $\theta-2 \theta$ scans of narrow regions around these four peaks are sufficient. The XRD analysis was possible on polished as well as on unpolished surfaces without any qualitative difference in the result.

\section{Results}

\subsection{Stress relief at $1200 \mathrm{~K}$}

For tungsten samples stress-relieved for 60 minutes at $1200 \mathrm{~K}$, the BSE images reveal a large number of small grains with typical dimensions of one up to a few microns (see Figure 1a). The outlines of these grains are not always perfectly clear due to the strong mechanical deformation introduced by the production process, and they show some shading in their greyscale contrast, which can be attributed to bending of grains or lattice distortions [13]. With the statistical analysis method described above, an average grain size of $<a_{B S E}>=1.17 \pm 0.03 \mu \mathrm{m}$ was derived from the BSE image (for the histogram see Figure 2). Using equation (2), an average grain boundary density of $\left\langle S_{V, B S E}\right\rangle=(1.42 \pm 0.03) \times 10^{6} \mathrm{~m}^{2} / \mathrm{m}^{3}$ was calculated (see Figure 4).

EBSD analysis yields a grain size $\left\langle a_{2^{\circ}}\right\rangle=1.86 \pm 0.02 \mu \mathrm{m}$ considering all grain boundaries with $\theta \geq 2^{\circ}$ (Figure 2), which corresponds to a density of these grain boundaries of 
$<S_{V, 1^{\circ}}>=(9.7 \pm 0.1) \times 10^{5} \mathrm{~m}^{2} / \mathrm{m}^{3}$ (Figure 4$)$. The reason that $<a_{2^{\circ}}>$ is larger than $\left\langle a_{B S E}>\right.$ can be attributed mainly to the fact that in BSE contrast also boundaries with $\theta<2^{\circ}$ can still be clearly identified. This results in a more fine-grained segmentation of the sample. Accordingly, while in the EBSD map for annealing temperature of $1200 \mathrm{~K}$ (see Figure 3a), also several grains with sizes above $5 \mu \mathrm{m}$ occur, these were not found in the corresponding BSE images (see histograms in Fig. 2). Also, the step width of the EBSD patterns was chosen at $0.5 \mu \mathrm{m}$ in order to be able to cover a large, statistically relevant surface area of the sample within a reasonable measurement time. In order to suppress random, isolated pixels that were indexed with a different orientation than their surroundings, only structures with a minimum size of $1 \mu \mathrm{m}^{2}$ (i.e., consisting of at least 4 pixels) were used for the grain size statistics. In the BSE image analysis, the point resolution is much better and allows also the identification of smaller grains. The scale length of zones delimited by high-angle boundaries is significantly larger than both $\left\langle a_{B S E}>\right.$ and $\left\langle a_{2^{\circ}}>\right.$, namely $\left\langle a_{10^{\circ}}>=3.10 \pm 0.11 \mu \mathrm{m}\right.$, which can be seen in the EBSD map in Figure $3 \mathrm{a}$ as well as in the corresponding histograms in Figure 2. This value of $<a_{10^{\circ}}>$ corresponds to a density of high-angle grain boundaries of $<S_{V, 10^{\circ}}>=(4.6 \pm 0.3) \times 10^{5} \mathrm{~m}^{2} / \mathrm{m}^{3}$. high-angle This demonstrates clearly that the grain sizes derived from BSE image analysis include low- and high-angle boundaries. The analysis of the misorientation histogram shows that more than half of the grain boundaries (54\%) are lowangle boundaries with $\theta<10^{\circ}$ (see Figure 5).

STEM images from such a sample show dense dislocation networks and tangles as well as many more or less ordered dislocation structures, which most likely correspond to low-angle grain boundaries (see Figure 1b). The total average dislocation density was determined to be $\rho_{\text {disloc }}=(3.2 \pm 1.7) \times 10^{14} \mathrm{~m} / \mathrm{m}^{3}$ (see also Figure 4). The dislocation density found here is significantly less than the dislocation density of $7.8 \times 10^{15} \mathrm{~m} / \mathrm{m}^{3}$ reported for tungsten filings that were produced at room temperature (i.e., cold-worked material) and analysed by Debye- 
Scherrer X-Ray diffraction [14]. Since our material was hot-rolled and also annealed at 1200 $\mathrm{K}$, we consider it reasonable that the observed dislocation density should be lower than in such cold-worked W. On the other hand, the dislocation density in our material coincides well with the value of $2.1 \times 10^{14} \mathrm{~m} / \mathrm{m}^{3}$ found in tungsten filings after annealing without recrystallization [14]. This is a similar heat treatment as for our samples, so we consider our observations to be well in agreement with [14].

\section{high-angle}

\subsection{Partial recrystallization at $1500 \mathrm{~K}$}

For partially recrystallized samples (30 minutes at $1500 \mathrm{~K})$, the outlines of some grains appear clearer in the BSE image, and these also show a very uniform greyscale contrast (see Figure 1c). This points towards the beginning of recrystallization, i.e., to the removal of distortion fields that were present in the initial sample. Overall, also the average grain size derived from BSE image analysis is now slightly larger at $\left\langle a_{B S E}>=1.54 \pm 0.02 \mu \mathrm{m}\right.$ (Figure 2). This corresponds to a grain boundary density of $\left\langle S_{V, B S E}\right\rangle=(1.03 \pm 0.01) \times 10^{6} \mathrm{~m}^{2} / \mathrm{m}^{3}$ (Figure 4). Yet, one can find also some particularly small grains. They can actually be seen best in the STEM images, e.g., approximately in the centre of Figure 1d. These are possibly grains that have freshly nucleated in the beginning of the recrystallization process. This heat treatment also leads to a significant decrease of the dislocation density to $\rho_{\text {disloc }}=(9.8 \pm 5.3) \times 10^{13} \mathrm{~m} / \mathrm{m}^{3}$ (Figure 4). Some grains, in particular also the small, freshly nucleated grains, are now practically free of dislocations, while others still contain dislocation networks comparable to those in the initial sample (see Figure 1d). Low-angle grain boundaries that can be resolved into their constituent dislocations are still present, but the amount of better ordered grain boundaries with possibly larger misorientation angles, which appear as more or less uniform dark bands in BF-STEM images (Figure 1d), increases noticeably. 
The EBSD analysis is actually not quite sensitive to this change of the grain boundary character. The misorientation histogram yields a fraction of low-angle grain boundaries with $\theta$ $<10^{\circ}$ of about $57 \%$, similar to the stress-relieved sample (Figure 5). The typical scale lengths $\left.<a_{2^{\circ}}\right\rangle=1.79 \pm 0.01 \mu \mathrm{m}$ and $\left\langle a_{10^{\circ}}\right\rangle=3.16 \pm 0.08 \mu \mathrm{m}$ are also very close to the values of the stress-relieved sample (Figure 2), and so are the corresponding grain boundary densities $<S_{V, 2^{\circ}}>=(1.01 \pm 0.01) \times 10^{6} \mathrm{~m}^{2} / \mathrm{m}^{3}$ and $\left\langle S_{V, 10^{\circ}}>=(4.9 \pm 0.2) \times 10^{5} \mathrm{~m}^{2} / \mathrm{m}^{3}\right.$ (Figure 4). However, in contrast to the stress-relieved sample, the grain boundary densities $<S_{V, B S E}>$ derived from BSE image analysis and $\left\langle S_{V, 2^{\circ}}>\right.$ from EBSD mapping for $\theta \geq 2^{\circ}$ are now much closer together (see Figure 4). Here, the limitations of our analysis with respect to the reliable identification of grain boundaries and the lateral resolution that were already explained in section 4.1 are obviously much less significant than for the sample annealed at $1200 \mathrm{~K}$.

The better agreement of BSE and EBSD grain sizes also sheds some more light onto the observation of more well-ordered grain boundaries in STEM images: It can be assumed that the ordering and densification of the cell boundaries in the partially recrystallized sample corresponds at least to some extent to recovery rather than actual nucleation and growth of new grains. Overall, the changes to the grain structure due to annealing for $30 \mathrm{~min}$ at $1500 \mathrm{~K}$ appear to be relatively subtle. The dislocation density appears to be the quantity most affected by this kind of heat treatment.

\subsection{Recrystallization and grain growth at $1700 \mathrm{~K}$}

For annealing temperatures of $1700 \mathrm{~K}$ and higher (with a holding time of 30 minutes), literature [9] predicts full recrystallization of the samples. One can also expect significant grain growth. Indeed, in BSE images of a sample annealed at $1700 \mathrm{~K}$ (Figure 1e), one finds many large grains with sizes of the order of $10 \mu \mathrm{m}$ and larger. They have clear outlines and a 
uniform BSE grayscale contrast. However, between these large grains, clusters of smaller grains with sizes in the micron range can still be found. Accordingly, the grain size distribution is now very broad (see Figure 2). Due to the small number of large grains in a typical image, it cannot be easily discerned if the distribution is, in fact, bimodal, or has just a large tail towards large grain sizes.

Practically all grains, both the small and large ones, show grooving at the grain boundaries. The larger grains show an apparently corrugated surface. This is presumably due to small grains that formed grooves at the grain boundaries after nucleation and a short, initial growth phase, but which were then consumed by other grains with a faster growth rate. Overall, the average grain size derived from BSE image analysis is now $\left\langle a_{B S E}>=2.92 \pm 0.13 \mu \mathrm{m}\right.$ (Figure 2). This is more than twice as large as for the stress-relieved sample. It corresponds to a grain boundary density of $<S_{V, B S E}>=(4.6 \pm 0.3) \times 10^{5} \mathrm{~m}^{2} / \mathrm{m}^{3}$ (Figure 4$)$. The impact of annealing at $1700 \mathrm{~K}$ on the dislocation density is even much stronger than on the grain size. Large areas of the sample are now practically free of dislocations (see Figure 1f). Only a few grains still contain strongly fragmented residues of the former dislocation network. Practically all of the easily resolvable low-angle grain boundaries that have been discussed for the lower annealing temperatures disappeared and were replaced by higher-angle grain boundaries. Overall, the dislocation density is now almost two orders of magnitude smaller than in the initial sample, namely $\rho_{\text {disloc }}=(5.2 \pm 3.0) \times 10^{12} \mathrm{~m} / \mathrm{m}^{3}$ (Figure 4).

For this sample, also the EBSD analysis of the grain boundaries shows significant changes. First of all, the fraction of low-angle grain boundaries with $\theta<10^{\circ}$ is now reduced to about 26\% (Figure 5). I.e., most grain boundaries are now, in fact, high-angle grain boundaries. Many grain boundaries are also annihilated due to grain growth, which is reflected by the overall smaller grain boundary density. The scale length of all grain boundaries with $\theta \geq 2^{\circ}$ is now $<a_{2^{\circ}}>=3.57 \pm 0.13 \mu \mathrm{m}$, corresponding to $<S_{V, 2^{\circ}}>=(4.1 \pm 0.2) \times 10^{5} \mathrm{~m}^{2} / \mathrm{m}^{3}$. This means that 
after annealing at $1700 \mathrm{~K}$ even the grains delimited by low-angle grain boundaries are larger than the grains enclosed by high-angle grain boundaries in the samples annealed at lower temperatures. The slight difference to the grain size $<a_{B S E}>$ from the BSE image analysis can, most likely, again be explained by the limited point resolution of the EBSD analysis, which leads to the suppression of grains smaller than $1 \mu \mathrm{m}^{2}$ (corresponding to less than four pixels) in the statistical analysis. The scale length of zones delimited by high-angle grain boundaries with $\theta \geq 10^{\circ}$ is still noticeably larger than both $\left\langle a_{B S E}>\right.$ and $\left\langle a_{2^{\circ}}\right\rangle$, with $\left\langle a_{10^{\circ}}\right\rangle=5.24 \pm 0.27 \mu \mathrm{m}$

(Figure 2). This leads to a correspondingly lower high-angle grain boundary density $<S_{V, 10^{\circ}}>=(2.8 \pm 0.2) \times 10^{5} \mathrm{~m}^{2} / \mathrm{m}^{3}$ (Figure 4$)$.

\subsection{Recrystallization and grain growth at $2000 \mathrm{~K}$}

After annealing a sample for $30 \mathrm{~min}$ at $2000 \mathrm{~K}$, even more grain growth is observed. This can be seen in the BSE image in Figure 1g, in the EBSD orientation map in Figure 3d as well as in the corresponding histograms in Figure 2. Grains with sizes of up to about $50 \mu \mathrm{m}$ can be found in the BSE image, and there are no clusters of small grains anymore, in contrast to the sample annealed at $1700 \mathrm{~K}$. Instead, smaller and larger grains are now more or less uniformly distributed across the sample. Each grain shows a uniform BSE contrast, and clear grooves are visible at the grain boundaries. The number of residual grooves inside large grains is now much smaller than at $1700 \mathrm{~K}$, most likely due to faster grain growth, which does not leave enough time for many small grains to stabilise and form grooves at their grain boundaries. BSE image analysis now yields an average grain size of $\left\langle a_{B S E}>=12.1 \pm 0.3 \mu \mathrm{m}\right.$ (histogram Figure 2), and a grain boundary density of $\left\langle S_{V, B S E}\right\rangle=(1.36 \pm 0.03) \times 10^{5} \mathrm{~m}^{2} / \mathrm{m}^{3}$ (Figure 4). Please note for the actual statistical analysis, an image with a larger field of view than that in Figure $1 \mathrm{~g}$ had to be used because of the large grain size. The image shown in Figure $1 \mathrm{~g}$ has 
the same magnification as Figures $1 \mathrm{a}, \mathrm{c}$ and e and is therefore better suited for visual comparison with the other samples. As for annealing at $1700 \mathrm{~K}$, the dislocation density is small. However, instead of occasional fragments of former dislocation networks, only some short, straight and mostly isolated dislocations were found (Figure 1h). The overall dislocation density is maybe even slightly smaller than for the sample annealed at $1700 \mathrm{~K}$, with a value of $\rho_{\text {disloc }}=(1.9 \pm 1.4) \times 10^{12} \mathrm{~m} / \mathrm{m}^{3}$ (Figure 4$)$.

For this type of sample, the fraction of low-angle grain boundaries with $2^{\circ} \leq \theta<10^{\circ}$ is now only $10 \%$ (Figure 5), so the vast majority of grains are now separated by high-angle boundaries. The small fraction of low-angle grain boundaries means also that the scale lengths for the whole ensemble of grain boundaries and that for the high-angle grain boundaries are only marginally different with $\left\langle a_{2^{\circ}}\right\rangle=11.1 \pm 0.3 \mu \mathrm{m}$ and $\left\langle a_{10^{\circ}}\right\rangle=12.7 \pm 0.5 \mu \mathrm{m}$ (Figure 2 ), which corresponds to $\left\langle S_{V, 2^{\circ}}\right\rangle=(1.48 \pm 0.05) \times 10^{5} \mathrm{~m}^{2} / \mathrm{m}^{3}$ and $\left\langle S_{V, 10^{\circ}}\right\rangle=(1.30 \pm 0.05) \times 10^{5} \mathrm{~m}^{2} / \mathrm{m}^{3}$, respectively (Figure 4). Accordingly, after annealing the tungsten sample at $2000 \mathrm{~K}$, the values from BSE image analysis and from EBSD, both for minimum misorientations of $2^{\circ}$ and $10^{\circ}$, are in reasonable agreement.

\subsection{Texture analysis}

From literature $[14,15,16,17]$, it is expected that the surface of hot-rolled tungsten plates is dominated by grains with their $\{100\}$ and $\{111\}$ planes parallel to the surface and their $<110>$ direction parallel to the rolling direction, i.e., with a $\{100\}<110>$ and $\{111\}<110>$ texture. A variation of the texture with depth is proposed, too $[15,18]$. Regarding the recrystallization texture, model predictions for bcc metals $[15,16,19,20]$ but also experimental results $[15$, $21,22]$ are somehow diverse: from sharpening the texture over rotating the texture, depleting different fractions of the texture (alpha vs. gamma fibre) to leaving the texture unchanged. In 
addition, recovery with selective grain growth versus recrystallization, i.e., nucleation of new grains and their growth is discussed in literature $[22,23]$. Therefore, we analysed the texture of the material after the annealing applied in this study.

By XRD we analysed a large number of polished and either stress-relieved or recrystallized ( $2000 \mathrm{~K})$ samples in addition to the four samples annealed at different temperatures that were investigated in detail with EBSD (section 4.1-4.4). The analyses confirm that always one surface of the stress-relieved specimens is dominated by the $\{100\}<110>$ and $\{111\}<110>$ textures (see [15]), which is clearly evident from Figure 3a-c. Surprisingly, on the reverse side of the $\mathrm{W}$ plates we always found a totally different texture. This one is dominated by $\{110\}$ planes parallel to the surface and $<211>$ or $<111>$ parallel to the rolling direction. We term the reverse side "B" side, while the front side with the expected texture will be called "A" side from here on. Overall, the texture on the "B" side exhibited somewhat more variation and was also weaker than that on the "A" side.

In order to evaluate the variation of the texture with depth, a cross-section of a stress-relieved, unpolished sample was additionally analysed with EBSD. Pole figures as usually obtained by XRD were calculated from the EBSD data (Fig. 6). The texture varies slightly throughout the cross-section with a drastic change in the last quarter of the $\sim 0.8 \mathrm{~mm}$ thick sheet close to the side with type "B" texture. In more detail, a thin layer with a more distorted texture exists on the " $\mathrm{A}$ " side, which is thinner than the layer that is typically removed by the polishing procedure $(\sim 100 \mu \mathrm{m})[3]$. Beyond that surface layer, the fraction of $\{111\}<110>$ increases slowly with depth, and the texture is quite sharp around the mid of the cross-section before it gets diffuse and less pronounced closer to the "B" side.

A possible explanation for the different texture of the two sides is that the plate from which the samples were cut by the manufacturer was rolled in a packet of two plates stacked on top of each other at least in the final rolling steps. This causes the "expected" texture of the " $\mathrm{A}$ " 
side to appear on the outside of the packet, i.e., on the side facing the rolling drums. On the inner side, where both plates touch each other, the texture observed on the "B" side is produced.

Annealing above $1200 \mathrm{~K}$ and recrystallization at $2000 \mathrm{~K}$ alter the texture of our samples, but the two different sides, "A" and "B", are still easily distinguishable with XRD and EBSD. The largest variation in the texture is observed between the samples annealed at 1500 and $1700 \mathrm{~K}$. The $\{111\}<110>$ component of the "A" side nearly vanishes after recrystallization at $2000 \mathrm{~K}$ (Figure 3d), while the dominant $<110>$ direction parallel to the rolling direction is altered, even though the $\{100\}<110>$ texture still dominates [16]. The reported double texture $\{100\}<011> \pm(12-16)^{\circ}$ for tungsten recrystallized at temperatures well above $2000 \mathrm{~K}[15,16$, 21] is not fully developed, but partially observed. The texture of the " $\mathrm{B}$ " side is overall weaker after recrystallization, i.e., all structures in the pole figure are smeared out.

On all four samples discussed in sections 4.1-4.4, the "A" side was polished and analysed. Figure 3 shows the orientation maps of the stress-relieved and the recrystallized $(2000 \mathrm{~K})$ specimen, which are used for the evaluation in 4.1-4.4. The dominance of $\{100\}$ and $\{111\}$ planes parallel to the surface is clearly visible (Figure $3 a$ and d), while the $<110>$ crystal direction is about parallel to the rolling direction (Figure $3 \mathrm{~b}$ and e). $\{110\}$ planes parallel to the surface are remarkably rare.

\section{Discussion}

After the heat treatments discussed here, one finds that the dislocation density is reduced strongly between annealing at 1200 and $1700 \mathrm{~K}$ and then stays at a low level of the order of a few $10^{12} \mathrm{~m} / \mathrm{m}^{3}$. On the other hand, the grain boundary density derived from BSE images continuously decreases with increasing annealing temperature (Figure 4). While annealing at 
$1200 \mathrm{~K}$ practically does not change the grain structure compared to the initial hot-rolled material, all recrystallization treatments were also accompanied by at least some grain growth. This corresponds to the decrease of the grain boundary density for higher temperatures (Figure 4).

Between the samples annealed at 1200 and $1500 \mathrm{~K}$, this is only observable in BSE images. The evaluation of the EBSD maps only includes grains with a minimum area of $1 \mu \mathrm{m}^{2}$ and a minimum misorientation of $2^{\circ}$. A higher spatial resolution was not easily attainable here while keeping the map area large enough for a good statistical evaluation, and the scatter of the orientations derived from the EBSD patterns by the analysis software did not allow setting a higher angular sensitivity. Due to these two cut-offs, the evaluation of EBSD data shows only minor differences between the two samples annealed at 1200 and $1500 \mathrm{~K}$ (see Figures 2 and 3). For both samples, more than half of the analysed grain boundaries have misorientations between $2^{\circ}$ and $10^{\circ}$ (see Figure 5), and are therefore considered as low-angle grain boundaries. In fact, it can be assumed that particularly in the sample annealed at $1200 \mathrm{~K}$, there is a substantial fraction of (sub)grain boundaries with misorientations even below $2^{\circ}$, which could not be reliably identified here by EBSD. The reduction of the density of such grain boundaries with very low misorientations after annealing at $1500 \mathrm{~K}$ is probably correlated to the reduction of the dislocation density (Figure 4). This is linked to the fact that, as described in section 3.2, dislocations in tangles and low-angle grain boundaries were intentionally counted together with other dislocations in the STEM image analysis. Still, subtle changes in the structure of grain boundaries are already visible in STEM images after annealing at 1500 $\mathrm{K}$ and most likely correspond to a recovery process, at least to some extent. In addition, small variations of the texture are observed. Overall, it can be stated that annealing at $1500 \mathrm{~K}$ leads to a reduction of the dislocation density, but does not affect the density of high-angle grain 
boundaries (and even of grain boundaries with $\theta \geq 2^{\circ}$ ) in comparison to a sample annealed at $1200 \mathrm{~K}$.

The significant grain growth due to annealing at 1700 and $2000 \mathrm{~K}$ is observed by BSE image evaluation as well as by EBSD analysis (see Figures 1, 2 and 3). As for the sample annealed at $1500 \mathrm{~K}$, the agreement between the grain boundary densities derived from BSE images and from EBSD maps including all grain boundaries with $\theta \geq 2^{\circ}$ is reasonable. This strengthens the assumption that the deviations observed for the sample annealed at $1200 \mathrm{~K}$ are indeed due to grain boundaries with misorientations $\theta<2^{\circ}$. These boundaries are mostly obliterated by annealing at $1500 \mathrm{~K}$ and higher. Annealing at $1700 \mathrm{~K}$ and higher also leads to an increased fraction of high-angle grain boundaries with misorientations of more than $10^{\circ}$ (see Figure 5). They comprise the majority of grain boundaries in these samples. Consequently, the grain sizes derived from BSE image analysis, and from EBSD (including either all grain boundaries with $\theta \geq 2^{\circ}$ or only those with $\theta \geq 10^{\circ}$ ) converge to very similar values for increasing annealing temperatures. The bottom line is that samples annealed at 1700 and $2000 \mathrm{~K}$ have similar, albeit very low dislocation densities, but significantly different densities of high-angle (and also low-angle) grain boundaries (Figure 4).

The behaviour with increasing annealing temperature of the tungsten samples investigated here can be easily understood by considering that one of the main driving forces for recrystallization is the latent energy stored in lattice deformation, i.e., dislocations [10]. The changeover to mostly high-angle grain boundaries reflects the fact that these are thermodynamically more stable.

The different evolutions of grain boundary and dislocation densities with the annealing temperature give the opportunity to compare the influence of grain boundary and dislocation density on other physical properties, e.g., the deuterium retention after plasma exposure $[5,6$, 
$7,8,24]$, since the samples recrystallized at 1700 and $2000 \mathrm{~K}$ have similar (very low) dislocation densities, but significantly different grain boundary densities. This is accompanied also by a small but measurable change of the texture. On the other hand, the samples annealed at 1200 and $1500 \mathrm{~K}$ have similar densities at least of high-angle grain boundaries, but different dislocation densities (Figure 4).

Considering the different fracture behaviour of low and high-angle grain boundaries that was found by Liu et al. [12], one can expect also the fracture behaviour of tungsten to change with recrystallization. Indeed, hot-rolled respectively stress-relieved tungsten shows at least some ductility. On the other hand, recrystallized tungsten, while still retaining some elastic flexibility, is extremely brittle. Experience from handling tungsten samples and wrapping foils from the annealing procedure confirms this. It can be expected that this also influences the behaviour of the tungsten samples with respect to, e.g., thermal shocks, or to the formation of gas-filled cavities (see, e.g., $[5,6])$ at grain boundaries due to bombardment with energetic hydrogen isotope ions.

\section{Summary}

After annealing at four different temperatures between 1200 and $2000 \mathrm{~K}$, the dislocation and grain boundary densities in rolled tungsten samples were determined by scanning electron microscopy and scanning transmission electron microscopy. It was found that, compared with a sample stress-relieved at $1200 \mathrm{~K}$, the dislocation density drops by about two orders of magnitude after recrystallization at $1700 \mathrm{~K}$ and stays at a similar, low value after recrystallization at $2000 \mathrm{~K}$. On the other hand, the density of high-angle grain boundaries is the same whether a sample is annealed at 1200 or $1500 \mathrm{~K}$, and continuously decreases with further increasing annealing temperature due to grain growth. Looking at the density of grain 
boundaries including those with very small misorientations that could only be clearly identified in BSE images, we found that this density starts to decrease already at $1500 \mathrm{~K}$. The grain boundary character also changes due to recrystallization. After annealing at 1200 and $1500 \mathrm{~K}$, more than $50 \%$ of the grain boundaries are low-angle grain boundaries with misorientations of less than $10^{\circ}$. After annealing at $1500 \mathrm{~K}$, signs of recovery and recrystallization can be found particularly in STEM images, but also in BSE images, while our EBSD analysis is apparently insensitive to these - still very subtle - changes. After annealing at 1700 or $2000 \mathrm{~K}$, the majority of grain boundaries are high-angle grain boundaries with at least $10^{\circ}$ of misorientation.

We also found that one rolling surface (which was investigated in detail here) of the stressrelieved samples used in this study exhibits a texture that is dominated by crystallographic planes close to $\{100\}$ and $\{111\}$ parallel to the surface, with the $<110>$ direction about parallel to the rolling direction. On the reverse side of the samples, we found mainly planes close to $\{110\}$ parallel to this surface. The $\{100\}<110>$ texture on the front side is more or less preserved upon recrystallization, while the $\{111\}<110>$ texture nearly vanishes. The texture on the reverse side weakens, but stays recognisable as well.

This extensive material characterisation can now be used as the basis for discussing, e.g., studies of deuterium retention in such tungsten samples after irradiation with low-temperature plasmas $[5,24]$.

\section{Acknowledgement:}

This work has been carried out within the framework of the EUROfusion Consortium and has received funding from the Euratom research and training programme 2014-2018 under grant agreement No 633053. The views and opinions expressed herein do not necessarily reflect those of the European Commission. 


\section{Literature}

[1] R. Frauenfelder, Vac. Sci. Technol. 6(1) (1969) 388-397

Solution and Diffusion of Hydrogen in Tungsten

http://dx.doi.org/10.1116/1.1492699

[2] J. Roth et al., J. Nucl. Mater. 390-391 (2009) 1-9

Recent Analysis of Key Plasma Wall Interactions Issues for ITER

http://dx.doi.org/10.1016/j.jnucmat.2009.01.037

[3] R.A. Causey, J. Nucl. Mater. 300 (2002) 91-117

Hydrogen Isotope Retention and Recycling in Fusion Reactor Plasma-Facing

Components

http://dx.doi.org/10.1016/S0022-3115(01)00732-2

[4] A. Manhard et al., Pract. Metallography 50(1) (2013) 5-16

A Step-By-Step Analysis of the Polishing Process for Tungsten Specimens http://dx.doi.org/10.3139/147.110215

[5] A. Manhard et al., J. Nucl. Mater 415(1S) (2011) S632-S635

Influence of the Microstructure on the Deuterium Retention in Tungsten http://dx.doi.org/10.1016/j.jnucmat.2010.10.045

[6] M. Balden et al., J. Nucl. Mater 414 (2011) 69-72

$D_{2}$ gas-filled blisters on deuterium-bombarded tungsten http://dx.doi.org/10.1016/j.jnucmat.2011.04.031

[7] M. Balden et al., J. Nucl. Mater 452 (2014) 248-256

Deuterium retention and morphological modifications of the surface in five grades of tungsten after deuterium plasma exposure

http://dx.doi.org/10.1016/j.jnucmat.2014.05.018

[8] M.H.J. ‘t Hoen et al., Nucl. Fusion 54 (2014) 083014 (10pp)

Surface morphology and deuterium retention of tungsten after low- and high-flux deuterium plasma exposure

http://dx.doi.org/10.1088/0029-5515/54/8/083014

[9] W. Espe: Werkstoffe der Hochvakuumtechnik (vol. 1), VEB Deutscher Verlag der Wissenschaften (1960) 
[10] R.E. Reed-Hill: Physical Metallurgy Principles (2 ${ }^{\text {nd }}$ ed.), D. Van Nostrand Company (1973)

[11] A. Jablonski et al., NIST SRD64 v. 3.2 (2010) National Institute of Standards and Technology, Gaithersburg, USA

[12] J. M Liu and B.-W. Shen, Acta Metall. 30(6) (1982), 1197-1202

Grain Boundary Fracture in Tungsten Bi-Crystals http://dx.doi.org/10.1016/0001-6160(82)90014-1

[13] S. Lindig et al., Phys.Scr. T145 (2011) 014039 (7pp) Sub-surface structures of ITER-grade W (Japan) and re-crystallized W after ITERsimilar low energy http://dx.doi.org/10.1088/0031-8949/2011/T145/014039

[14] G.K. Williamson and R.E. Smallman, Philos. Mag. 1(1) (1956) 34-46 III. Dislocation Densities in some Annealed and Cold-Worked Metals from Measurements on the X-Ray Debye-Scherrer Spectrum http://dx.doi.org/10.1080/14786435608238074

[15] D. Raabe, B. Mülders, G. Gottstein and K- Lücke, Materials Science Forum 157-162 (1994) 841-846 Textures of Cold Rolled and Annealed Tantalum http://dx.doi.org/ 10.4028/www.scientific.net/MSF.157-162.841

[16] Y.B. Park, D.N. Lee and G. Gottstein, Mater.Sci. Eng. A257 (1998) 178-84 A Model of the Development of Recrystallization Textures in Body Centered Cubic Metals http://dx.doi.org/10.1016/S0921-5093(98)00837-5

[17] Y.B. Park, D.N. Lee and G. Gottstein, Acta Mater. 46 (1998) 3371-9 The Evolution of Recrystallization Textures in Body Centered Cubic Metals http://dx.doi.org/10.1016/S1359-6454(98)00052-4

[18] F.J. Humphtreys and M Hatherly, Recrystallization and Related Annealing Phenomena, Elsevier (Amsterdam), 2004 
[19] D.N. Lee, Metals and Materials 5 (1999) 401-17

Strain Energy Release Maximization Model for Recrystallization Textures http://dx.doi.org/10.1007/BF03026153

[20] D.N. Lee, Inter. J. Mechanical Science 42 (2000) 1645-78

Strain Energy Release Maximization Model for Evolution of Recrystallization Textures http://dx.doi.org/10.1016/S0020-7403(99)00095-8

[21] J.W. Pugh, Transaction of the Metallurgical Society of AIME, 212 (1958) 637-663

The Temperature Dependence of Preferred Orientation in Rolled Tungsten

[22] J. Reiser, M. Rieth, B. Dafferner, A. Hoffmann, X. Yi and D.E.J. Armstrong, J. Nucl. Mater. 424 (2012) 197-203

Tungsten Foil Laminate for Structural Divertor Applications - Analyses and Characterisation of Tungsten Foil http://dx.doi.org/10.1016/j.jnucmat.2012.02.030

[23] D.N. Lee, J. Mater. Processing Technol. 117 (2001) 307-310

A Stability Criterion for Deformation and Deposition Textures of Metals During Annealing http://dx.doi.org/10.1016/S0924-0136(01)00787-7

[24] A. Manhard, PhD thesis, Augsburg University (2012) Deuterium Inventory in Tungsten after Plasma Exposure: A Microstructural Survey http://opus.bibliothek.uni-augsburg.de/opus4/frontdoor/index/index/docId/1981 


\section{Figures}
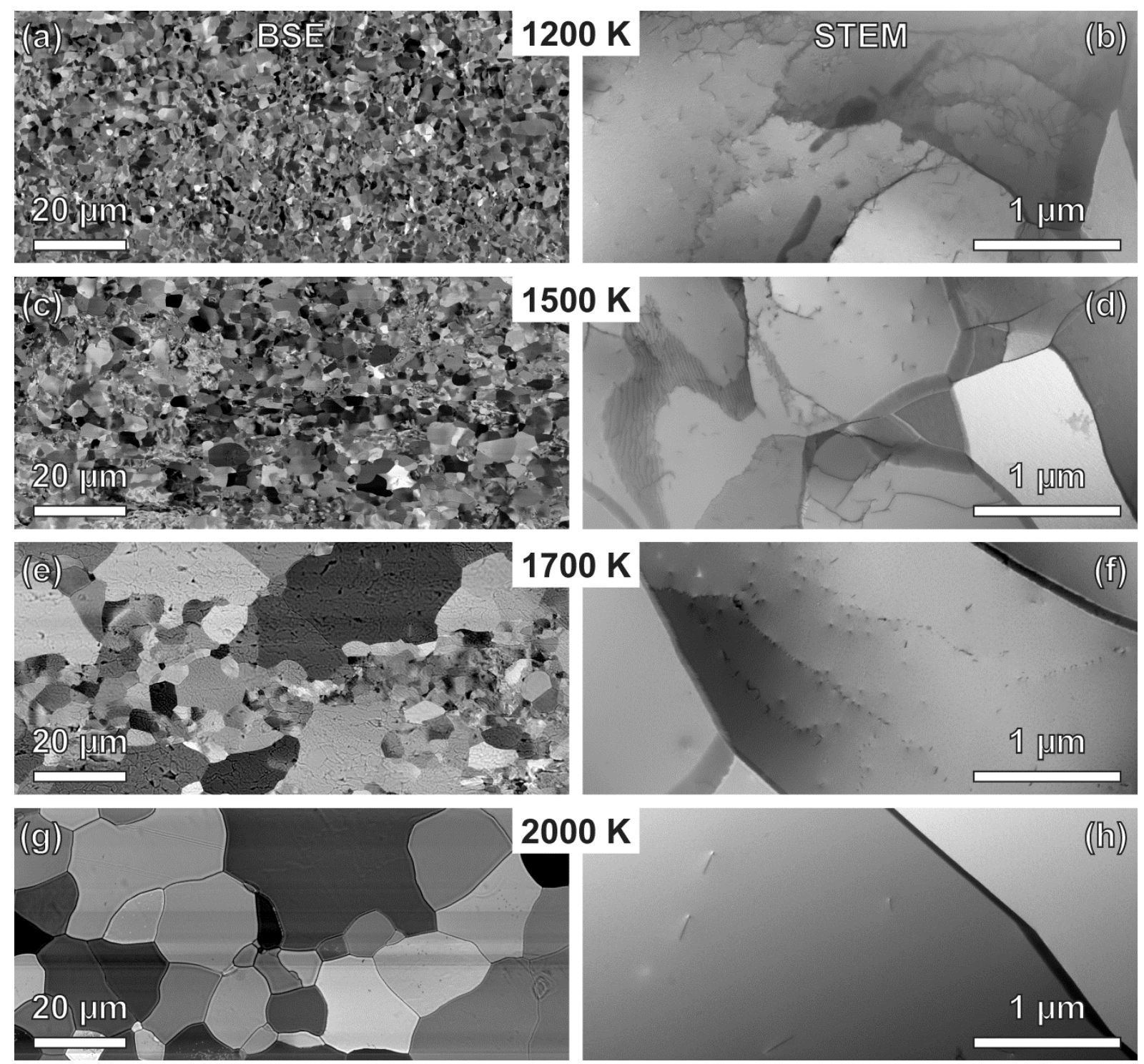

Figure 1 (two columns): Panels $a, c$, $e$ and $g$ show BSE images of $W$ annealed at 1200, 1500, 1700 and $2000 \mathrm{~K}$, respectively. Both small and high-angle grain boundaries are visible, and the greyscale contrast is not a direct measure for the misorientation angle. Panels $b, d, f$ and h show BF-STEM images of W samples annealed at the same temperatures. Clear, dark (and sometimes tangled) lines correspond to dislocations, whereas broader dark bands are grain boundaries. Occasional faint, blurry and often irregular shapes are due to unavoidable contamination on the samples. 


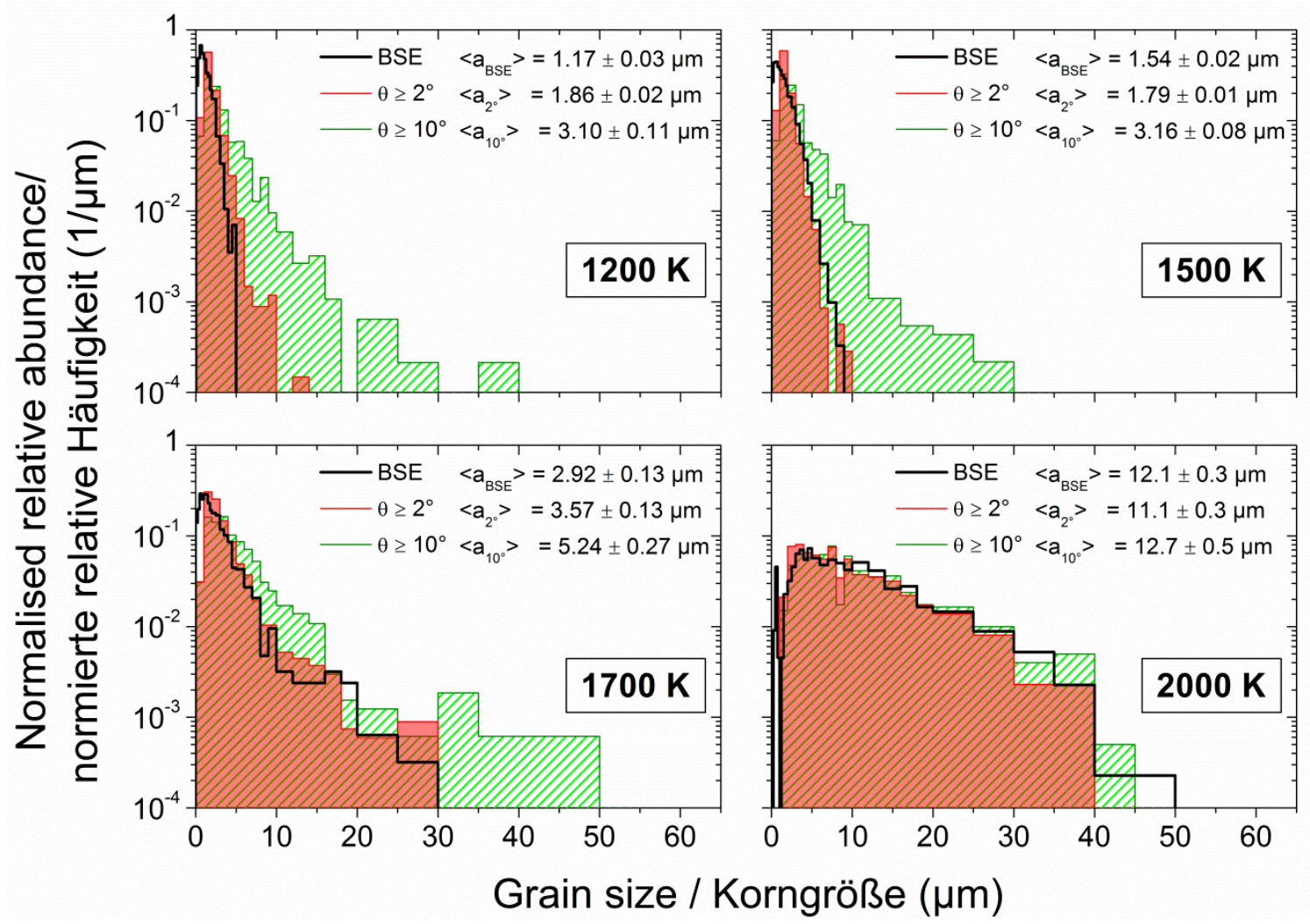

Figure 2 (two columns): Grain size statistics (i.e., equivalent square side length) for tungsten samples annealed at 1200, 1500, 1700 and $2000 \mathrm{~K}$. The histograms are derived from BSE image analysis as well as from EBSD mapping. EBSD grain sizes are shown both for a minimum misorientation of $2^{\circ}$ and $10^{\circ}$. For all histograms, the relative abundances have been normalised so they approximate the probability density function of the grain size. 

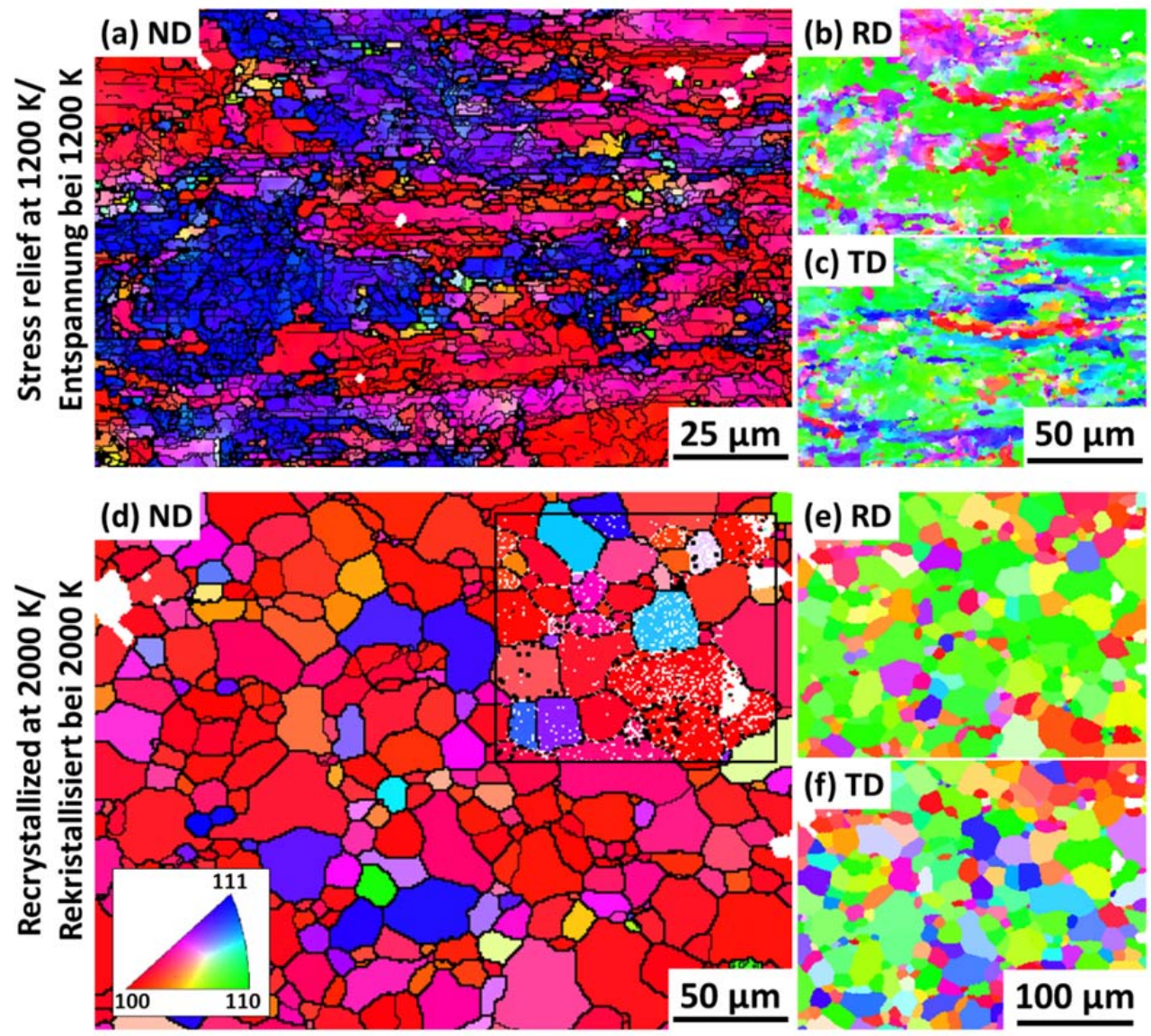

Figure 3 (two columns): EBSD orientation maps of the stress-relieved sample at $1200 \mathrm{~K}$ (top: $a-c)$ and the recrystallized sample at $2000 \mathrm{~K}$ (bottom: $d-f$ ). The orientations are colourcoded in respective to the normal $N D(a, d)$, rolling $R D(b, e)$ and transverse direction $T D(c$, f). The legend of the colour code is given in $d$. Note that the magnification differs by a factor two between the maps in the left $(a, d)$ and in the right $(b, c, e, f)$ column. The insert in the upper right in $d$ shows the data without interpolation (for details see text). Furthermore, the grain boundaries with a misorientation angle $>2^{\circ}$ (thin black lines) and $>10^{\circ}$ (thick black lines) are marked in a and $d$. 


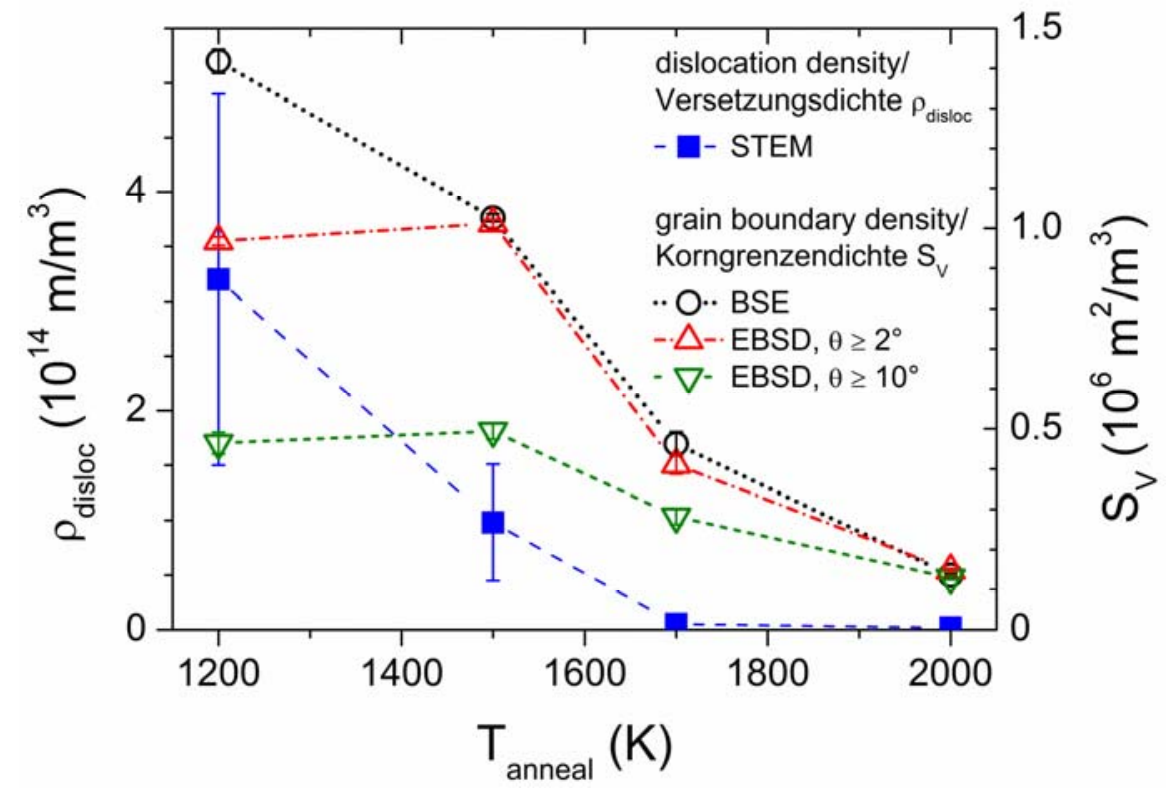

Figure 4 (one column): Dislocation densities $\rho_{\text {disloc }}$ (left y-axis) and grain boundary densities $\left.<S_{V}\right\rangle$ (right $y$-axis) in tungsten samples depending on the annealing temperature. The circles denote the values based on BSE image evaluation. The up and down triangles represent grain boundaries measured by EBSD with minimum misorientations of $2^{\circ}$ and $10^{\circ}$, respectively.

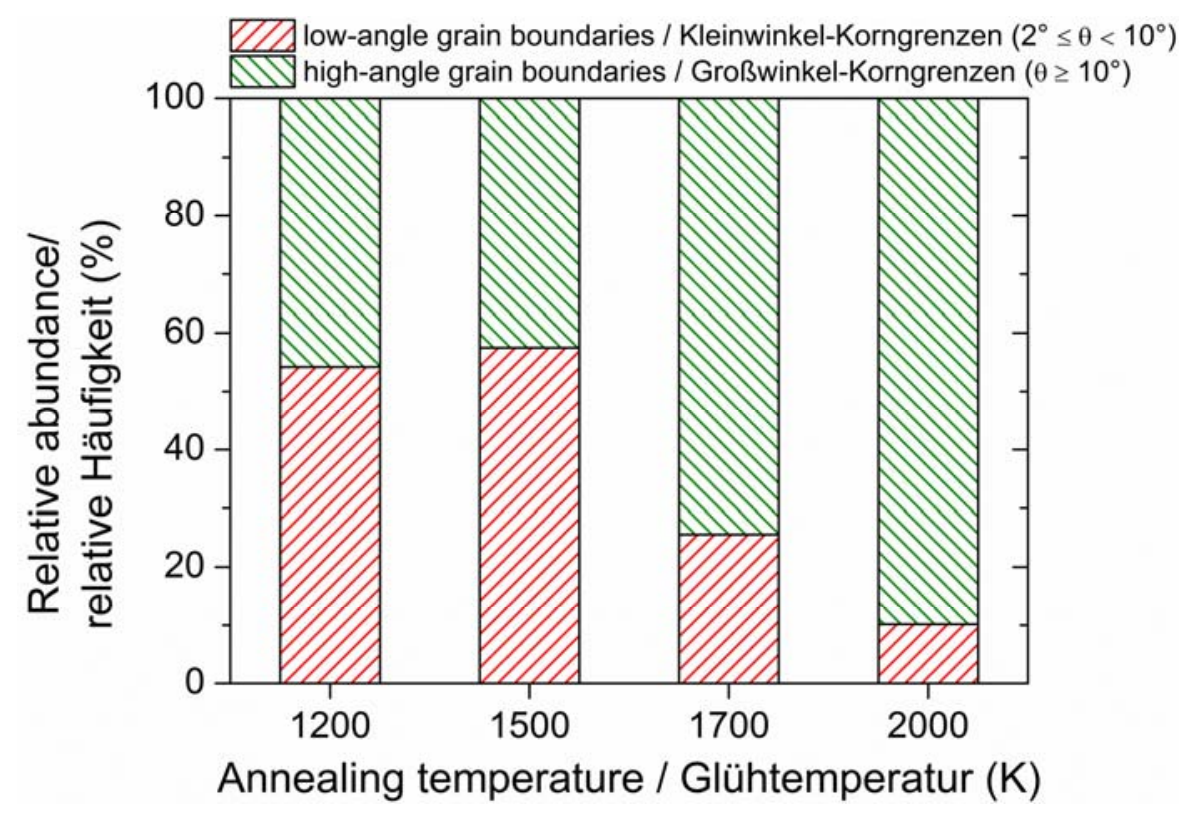

Figure 5 (one column): Abundance of low-angle $\left(2^{\circ} \leq \theta<10^{\circ}\right)$ and high-angle $\left(\theta \geq 10^{\circ}\right)$ grain boundaries in tungsten depending on the annealing temperature. 


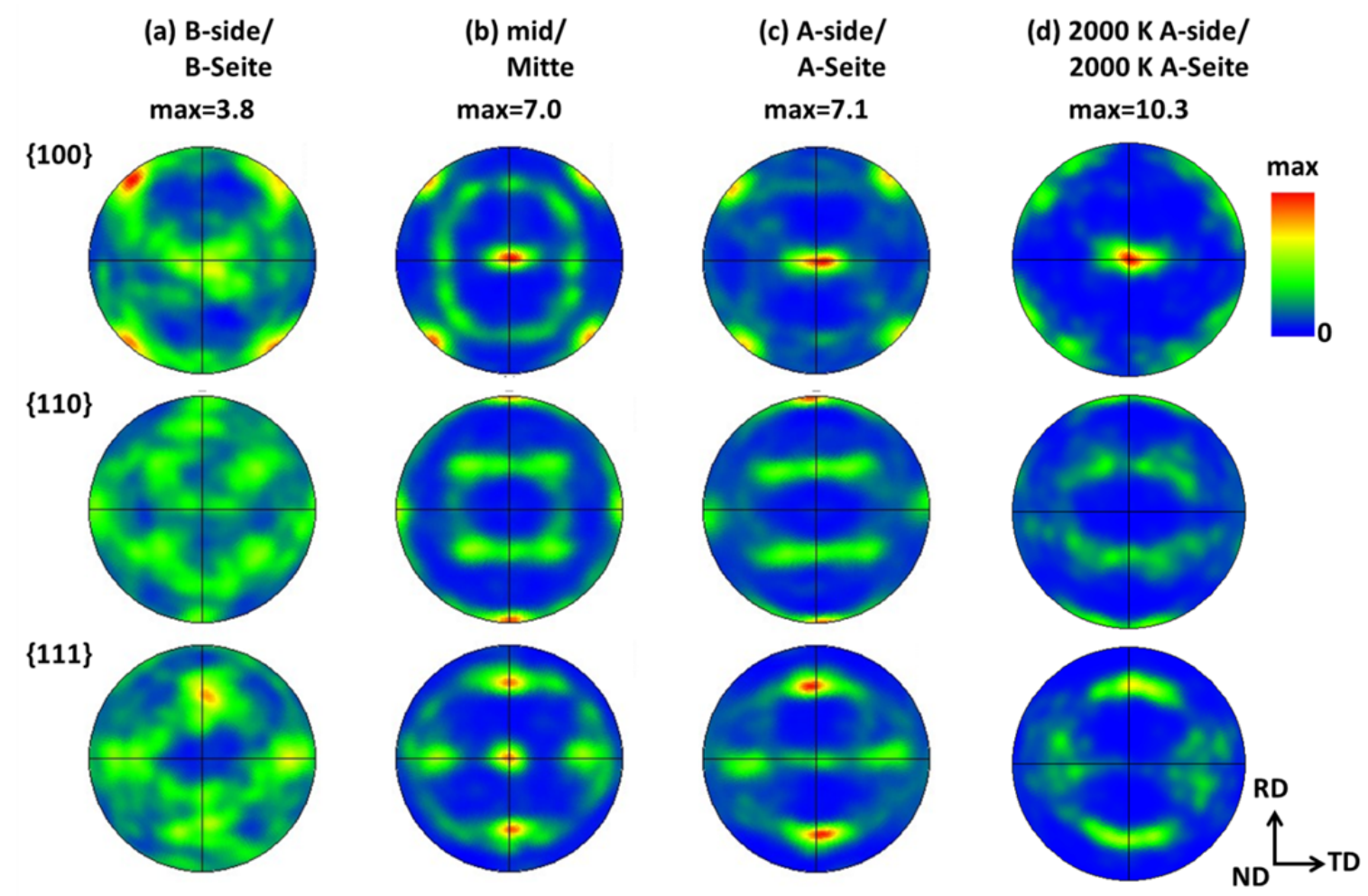

Figure 6 (two column): Pole figures from EBSD texture analyses of the cross-section of a $1200 \mathrm{~K}$ annealed sample (a) at $100 \mu \mathrm{m}$ from B-side, (b) in the middle of the sample and (c) at $100 \mu \mathrm{m}$ below the A-side. For comparison, the pole figures of the A-side surface of the sample annealed at $2000 \mathrm{~K}(\mathrm{~d})$ are also shown (corresponding orientation maps in figure 3 $(d-f))$. 